\title{
Illustrations or Graphs: Some Students Benefit from One over the Other
}

\author{
Michael Lipschultz and Diane Litman \\ Computer Science Department, University of Pittsburgh \\ \{lipschultz, litman\}@cs.pitt.edu
}

\begin{abstract}
We examine whether there are differences between students regarding the utility of learning from visual representations (illustrations or graphs) within the context of a typed natural language-based conceptual physics tutoring system. Showing half of the students only illustrations and the other half only graphs, we found that novices benefited from illustrations, whereas non-novices showed no difference.
\end{abstract}

Keywords: ITS, graphics, dialogue, conceptual physics, student modeling.

\section{Introduction}

1-on-1 human tutoring is a very effective method of instruction 8 . Intelligent tutoring systems (ITSs) have been developed to provide similar, but computerbased, tutoring; they too are effective at improving student knowledge [11. ITSs use various representations to convey information, such as through natural language (NL) or through visuals. Our ITS presents visuals within the context of a NL-based tutoring system. Other systems using both tend to present them together. The NL representation may be expository with the visuals showing the concepts being explained [1] or may be more interactive in the form of a dialogue accompanied by a static image [5] or an interactive simulation [4]. These systems present only one kind of visual, which may not be best for students [79.

Research suggests novices will benefit from concrete illustrations because they are relatable for those students [7. Non-novices will benefit more from abstract representations, such as graphs, because the concepts are presented without problem-specific context and so are easier to learn in a context-free way [7. Therefore, we believe that adapting visual representations to students will improve learning. Some ITSs make use of multiple visual representations, but do not adapt representation selection to learners [10.

We present here the first step towards adapting visuals to learners, by showing either illustrations or graphs during the course of typed dialogue conceptual physics tutoring. We hypothesize novices will learn more when seeing illustrations over graphs during tutoring (hypothesis H1) and that skilled students will learn more when seeing graphs during tutoring over illustrations (hypothesis $\mathrm{H} 2$ ). We find evidence supporting H1, but not for H2. 
Problem Statement: A kangaroo can jump about $1.50 \mathrm{~m}$ straight up. What is the magnitude of the take-off velocity?

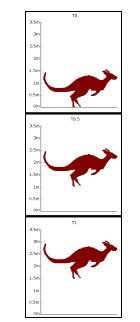

(a) Illustration

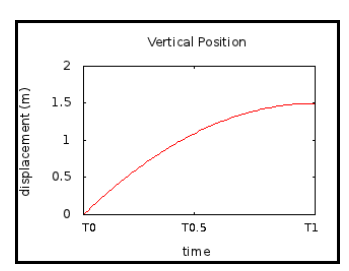

(b) Graph
Tutor $_{1}$ : The figure shown represents the kangaroo's position in the vertical direction. The $\mathrm{x}$-axis is time and the $\mathrm{y}$ axis is vertical position. At what time was the kangaroo's velocity greatest? Student $_{1}$ : at T1 (top of jump) Tutor $_{2}$ : I don't think that's right. The kangaroo is moving fastest when it first takes off (at T0). We can see this in the figure. Velocity is the change in position over the change in time. So let's take a look at the change in position at three instances during the jump: beginning, middle, and end.

(c) Start of 1st reflection dialogue

Fig. 1. The first tutoring problem. The problem statement is at the top. Subfigures 1a and $1 \mathrm{~b}$ show the basic visuals for each condition. Subfigure $1 \mathrm{c}$ shows the beginning of the first reflection dialogue.

\section{Methods}

The experiment compared two conditions: one where students saw only illustrations during tutoring and the other where students saw only graphs. Tutoring consisted of 2 problems and 3 reflection questions per problem, within the Rimac tutoring system [6], which consists of a problem-solving component (Andes [1] ) and a post-problem discussion component.

29 college students without college physics were recruited and randomly assigned to one of the conditions. Students in both conditions filled out a background survey, completed the Paper Folding Test (PFT, a standard spatial reasoning test [3]), and read a short text on kinematic physics (the domain tutored).

Students took a pretest (one of two counterbalanced isomorphic tests), consisting of 31 multiple choice questions, to measure their incoming physics knowledge. 5 questions were problem-solving or numeric and 26 were conceptual questions. Of the conceptual questions, 8 did not include visuals, 9 involved illustrations, and 9 involved graphs (graph and illustration questions were isomorphic). From these, we have five measures of learning: overall, problemsolving, conceptual, score-illustration, and score-graph.

We trained students to use Rimac, then began tutoring. With the help of a walkthrough dialogue, students first solved a physics problem in Andes [11. Figure 1 shows the first problem statement. After solving the problem, they began the reflection dialogue, where they reflected on concepts involved in the problem. During this dialogue, up to 7 visuals relevant to each student's condition are shown to help explain concepts (modified versions of Figures 1a, 1b). Figure $1 \mathrm{c}$ shows the start of a reflection dialogue. After completing the last reflection 
dialogue, they repeat for another problem and three reflection dialogues. Both problems and all six reflection questions were approved by physics teachers. At the end, students took a post-test.

\section{Results}

T-tests confirmed conditions were balanced on pretest score $(\mathrm{p}=0.943)$ and PFT $(\mathrm{p}=0.524)$. Based on [7, we believed PFT should correlate with score-graph on the pretest but not score-illustration on the pretest. Both correlate (p-values 0.033 and 0.023 ), suggesting PFT may not measure the spatial reasoning used.

Of the 29 students who participated in the study, 7 did not show learning gains, 5 in the illustration condition and 2 in the graphs. In the following analysis, we consider only the 22 students who had learning gains (including all 29 gave similar, but not significant or trend patterns).

We ran 5 ANCOVAs (1 for each measure of learning) to identify main and interaction effects. For each, the dependent variable was the post-test score, the covariate was the pre-test score, and the independent variables were condition (illustration or graph) and overall pretest score (median split: high or low). Although no main effects, there was a condition-pretest interaction effect for all ANCOVAs, except problem-solving, see Table 1.

$\mathrm{H} 1$ is confirmed. For each of the four significant interactions, low pretesters who saw illustrations scored higher than low pretesters seeing graphs.

$\mathrm{H} 2$ is not supported. For overall, conceptual, and score-graph, those who saw illustrations scored higher than those who saw graphs. For score-illustration, those who saw graphs scored higher than those who saw illustrations. Comparing this to score-graph, we see that during tutoring better performance on scoregraph came from those who saw illustrations and better performance on scoreillustration came from those who saw graphs.

Table 1. Pretest score and condition interactions. Cells contain the adjusted post-test scores (percentages out of the total number of questions for that subset of the test, e.g. out of nine for graphs) from the ANCOVAs, with $95 \%$ confidence intervals beneath.

\begin{tabular}{|l|c|c|c|c|c|}
\hline Test & \multicolumn{2}{|c|}{ Pretest=High } & \multicolumn{2}{c|}{ Pretest=Low } & Signif. \\
& Illus. & Graph & Illus. & Graph & Interaction? \\
\hline $\mathrm{N}$ & 2 & 4 & 7 & 9 & \\
\hline \multirow{2}{*}{ Overall } & 0.876 & 0.865 & 0.784 & 0.655 & $\mathrm{Y}$ \\
& $(0.647,1.106)$ & $(0.662,1.069)$ & $(0.632,0.935)$ & $(0.555,0.755)$ & \\
\hline \multirow{2}{*}{ P Solving } & 0.815 & 0.550 & 0.525 & 0.544 & $\mathrm{~N}$ \\
& $(0.476,1.166)$ & $(0.474,1.108)$ & $(0.376,0.910)$ & $(0.307,0.619)$ & \\
\hline \multirow{2}{*}{ Conceptual } & 0.876 & 0.870 & 0.817 & 0.694 & $\mathrm{Y}$ \\
& $(0.674,1.078)$ & $(0.695,1.045)$ & $(0.691,0.943)$ & $(0.604,0.784)$ & \\
\hline \multirow{2}{*}{ Graphs } & 0.828 & 0.785 & 0.801 & 0.634 & $\mathrm{Y}$ \\
& $(0.656,1.001)$ & $(0.614,0.957)$ & $(0.690,0.911)$ & $(0.550,0.718)$ & \\
\hline \multirow{2}{*}{ Illustrations } & 0.878 & 0.916 & 0.798 & 0.702 & $\mathrm{Y}$ \\
& $(0.630,1.127)$ & $(0.709,1.124)$ & $(0.650,0.946)$ & $(0.582,0.821)$ & \\
\hline
\end{tabular}




\section{Conclusions and Future Work}

Half of the students saw only illustrations and the other half only graphs within a NL-based conceptual physics ITS. We found that novices benefit from illustrations, but no difference existed for non-novices. Therefore, non-novices might benefit from seeing both representations, alternated according to a schedule, which others have found helps learning 10 .

We are now developing a student model for predicting which visual is more beneficial using features found useful in similar tasks [9/2/7 that were collected in this pilot study. With a student model, we plan on evaluating whether an adaptive tutoring system shows greater learning gains than a non-adaptive one in another study.

Acknowledgments. We thank Wenting Xiong, Huy Viet Nguyen, the rest of the Itspoke team, the Rimac team, Jingtao Wang, and Vincent Aleven for their contributions. This research was supported by IES Grant R305A10063 to the University of Pittsburgh. The opinions expressed are those of the authors and do not represent the views of IES or the U.S. DoE.

\section{References}

1. Albacete, P.L., VanLehn, K.: Evaluation the effectiveness of a cognitive tutor for fundamental physics concepts. In: Proc. Cog. Sci. Society (2000)

2. Chi, M., VanLehn, K., Litman, D.: Do micro-level tutorial decisions matter: applying reinforcement learning to induce pedagogical tutorial tactics. In: Aleven, V., Kay, J., Mostow, J. (eds.) ITS 2010, Part I. LNCS, vol. 6094, pp. 224-234. Springer, Heidelberg (2010)

3. Ekstrom, R., French, J., Harman, H.: Manual for kit of factor referenced cognitive tests. Educational Testing Service, Princeton (1976)

4. Graesser, A.C., Chipman, P., Haynes, B.C., Olney, A.: AutoTutor: an intelligent tutoring system with mixed-initiative dialogue. IEEE Trans. Educ., 612-618 (2005)

5. Graesser, A.C., Lu, S., Jackson, G.T., Mitchell, H.H., Ventura, M., Olney, A., Louwerse, M.M.: AutoTutor: A tutor with dialogue in natural language. Behavior Research Methods 36(2), 180-192 (2004)

6. Katz, S., Jordan, P., Litman, D., The Rimac Project Team: Rimac: A naturallanguage dialogue system that engages students in deep reasoning. SREE (2011)

7. Kozhevnikov, M., Motes, M.A., Hegarty, M.: Spatial visualization in physics problem solving. Cognitive Science 31(4), 549-579 (2007)

8. Kulik, C.L.C., Kulik, J.A., Bangert-Drowns, R.L.: Effectiveness of mastery learning programs: A meta-analysis. Review of Educational Research 60(2), 265-299 (1990)

9. Meltzer, D.E.: Relation between students problem-solving performance and representational format. Am. J. Phys. 73, 463 (2005)

10. Rau, M., Rummel, N., Aleven, V., Pacilio, L., Tunc-Pekkan, Z.: How to schedule multiple graphical representations? A classroom experiment with an intelligent tutoring system for fractions. In: ICLS, pp. 64-71 (2012)

11. VanLehn, K., Lynch, C., Schulze, K., Shapiro, J., Shelby, R., Taylor, L., Treacy, D., Weinstein, A., Wintersgill, M.: The Andes physics tutoring system: Lessons learned. IJAIED, 147-204 (2005) 